\title{
Cost comparison of surgical management of nonsagittal synostosis: traditional open versus endoscope-assisted techniques
}

\author{
Ema Zubovic, MD, ${ }^{1}$ Jodi B. Lapidus, MD, MPHS, ${ }^{2}$ Gary B. Skolnick, BS, ${ }^{1}$ \\ Sybill D. Naidoo, PhD, RN, CPNP, Matthew D. Smyth, MD, ${ }^{3}$ and Kamlesh B. Patel, MD, MSc ${ }^{1}$ \\ 1Division of Plastic and Reconstructive Surgery, Department of Surgery, Washington University School of Medicine in St. Louis, \\ Missouri; '2Division of Plastic and Reconstructive Surgery, Department of Surgery, University of California, San Francisco, \\ California; and ${ }^{3}$ Department of Neurosurgery, Washington University School of Medicine in St. Louis, Missouri
}

\begin{abstract}
OBJECTIVE Management of craniosynostosis at an early age is important for mitigating the risk of abnormal cranial development, but treatment can result in significant expenses. Previous research has shown that endoscope-assisted craniectomy (EAC) is less costly than open cranial vault remodeling (CVR) for patients with sagittal synostosis. The aim of this study was to strengthen the existing body of healthcare cost research by elucidating the charges associated with open and endoscopic treatment for patients with nonsagittal synostosis.
\end{abstract}

METHODS The authors performed a retrospective analysis of data obtained in 41 patients who underwent open CVR and 38 who underwent EAC with postoperative helmet therapy for nonsagittal, single-suture craniosynostosis (metopic, coronal, and lambdoid) between 2008 and 2018. All patients were < 1 year of age at the time of surgery and had a minimum 1 year of follow-up. Inpatient charges, physician fees, helmet charges, and outpatient clinic visits in the 1 st year were analyzed.

RESULTS The mean ages of the children treated with EAC and open CVR were 3.5 months and 8.7 months, respectively. Patients undergoing EAC with postoperative helmet therapy required more outpatient clinic visits in the 1 st year than patients undergoing CVR (4 vs 2; $p<0.001$ ). Overall, $13 \%$ of patients in the EAC group required 1 helmet, $30 \%$ required 2 helmets, $40 \%$ required 3 helmets, and $13 \%$ required 4 or more helmets; the mean total helmeting charges were $\$ 10,072$. The total charges of treatment, including inpatient charges, physician fees, outpatient clinic visit costs, and helmet charges, were significantly lower for the EAC group than they were for the open CVR group $(\$ 50,840$ vs $\$ 95,588$; $p<0.001)$.

CONCLUSIONS Despite the additional charges for postoperative helmet therapy and the more frequent outpatient visits, EAC is significantly less expensive than open CVR for patients with metopic, coronal, and lambdoid craniosynostosis. In conjunction with the existing literature on clinical outcomes and perioperative resource utilization, these data support EAC as a cost-minimizing treatment for eligible patients with nonsagittal synostosis.

https://thejns.org/doi/abs/10.3171/2019.11.PEDS19515

KEYWORDS craniosynostosis; cost; endoscopic; cranial vault remodeling; fronto-orbital advancement; craniofacial

$\mathrm{M}$ INIMALLY invasive techniques for the treatment of all forms of single-suture craniosynostosis have gained in popularity since the introduction by Jimenez and Barone of strip craniectomy for sagittal synostosis in $1998 .{ }^{17}$ Endoscope-assisted craniectomy (EAC) has since also been employed in the treatment of metopic, coronal, and lambdoid suture synostoses. Epidemiological studies have shown changes in the relative incidence of these diagnoses from previously accepted figures. ${ }^{22,33}$ Sagittal suture synostosis remains the most common form, but the most recent data indicate a rise in metopic synostosis, now regarded as the second most common type

ABBREVIATIONS CPT = Current Procedural Terminology; CVR = cranial vault remodeling; $E A C=$ endoscope-assisted craniectomy; FOA = fronto-orbital advancement; LOS = length of stay.

SUBMITTED September 5, 2019. ACCEPTED November 6, 2019.

INCLUDE WHEN CITING Published online January 10, 2020; DOI: 10.3171/2019.11.PEDS19515. 
of craniosynostosis, followed by unicoronal and unilateral lambdoid synostosis.,37,38 Together, these three nonsagittal single-suture fusions account for approximately $40 \%$ of patients with nonsyndromic craniosynostosis. ${ }^{22}$

Traditional open treatment for metopic and unicoronal synostosis is fronto-orbital advancement (FOA). ${ }^{26}$ Lambdoid synostosis is classically treated by posterior cranial vault remodeling (CVR). ${ }^{9}$ These open techniques are commonly and often exclusively practiced in craniofacial centers around the world. Endoscopic techniques combined with postoperative molding helmet therapy have shown promising postoperative outcomes, with the benefits of reductions of the following: blood loss, transfusion requirement, operative time, and duration of hospitalization. 1,6,10,13,14,18,21,31,39 The endoscopic approach is becoming an increasingly appealing alternative to traditional open reconstructions. Concurrently, there has been increasing interest in the discrepancy in the costs of these two significantly different surgical approaches to the same cranial malformation. Multiple studies have investigated this question for the treatment of sagittal synostosis, ${ }^{1,31,39}$ but the literature addressing financial factors for the major forms of nonsagittal synostosis is lacking.

The primary aim of this study was to compare the charges for the 1st year of treatment for EAC with helmet therapy versus traditional open CVR for single-suture metopic, coronal, and lambdoid synostoses. The secondary aim was to describe the trends in treatment charges over time. We included all measurable charges in the 1st year of treatment, including perioperative hospitalization, physician fees, outpatient follow-up, and helmet therapy for the endoscopic cohort.

\section{Methods}

\section{Study Design}

This was a retrospective case-control study of patients treated at a single institution (St. Louis Children's Hospital, St. Louis, Missouri) over a 10-year period (2008-2018) by 3 plastic surgeons and 1 neurosurgeon for repair of single-suture metopic, coronal, and lambdoid synostoses. The study was approved by the Institutional Review Board of Washington University School of Medicine in St. Louis.

\section{Patient Selection}

All patients treated for nonsagittal craniosynostosis with either CVR or EAC between 2008 and 2018 were reviewed for eligibility. Patients treated at $<1$ year of age for nonsyndromic single-suture nonsagittal synostosis (metopic, unicoronal, lambdoid), with a minimum of 1 year of clinical follow-up, were included in the study. Patients with syndromic or multisuture synostosis, inadequate follow-up, helmet therapy noncompliance, or lack of complete financial data were excluded from the study.

\section{Treatment Protocol and Technique}

The standard protocol at our institution for evaluation of children with craniosynostosis includes multidisciplinary preoperative evaluation by a craniofacial surgeon and a pediatric neurosurgeon, typically with 3D CT imaging to confirm the diagnosis of craniosynostosis, using a low-dose radiation protocol. We offer both open and endoscope-assisted repairs to eligible patients presenting before 6 months of age (2-4 months preferred for coronal synostosis) and only open CVR to patients presenting for surgery after 6 months. In patients younger than 6 months of age, the decision to proceed with EAC versus open CVR is multifactorial and takes into account family preference and the ability to comply with postoperative helmet therapy. Our preferred approach for EAC in metopic synostosis has been previously published. ${ }^{29}$ For coronal and lambdoid synostosis, we perform similar strip craniectomies along the fused suture line, $1-1.5 \mathrm{~cm}$ wide, ensuring lateral extension to the level of the squamosal suture for patients with coronal synostosis. Open CVR is performed using standard FOA techniques for metopic or unicoronal synostosis, and patients with lambdoid synostosis are treated with posterior CVR..$^{25,30,34}$ For open CVR, we use a resorbable plating system (SonicWeld, KLS Martin). Patients treated with open CVR are admitted to the pediatric ICU for 1 night postoperatively, with transfer to a regular surgical floor on postoperative day 1 . A closedsuction drain is placed subcutaneously and removed prior to discharge. Tapered doses of intravenous dexamethasone are given to minimize edema in all patients undergoing open CVR, beginning with an intraoperative dose.

Patients treated with EAC are admitted to a regular surgical floor postoperatively, and typically discharged home on postoperative day 1. Patients see the orthotist a few days prior to surgery for consultation and scanning for the helmet, and helmet therapy is typically initiated within 1-3 days postoperatively. Local orthotists fit patients with their first helmet in the hospital prior to discharge on postoperative day 1, and patients using more distant orthotic companies have an appointment within the first 3 postoperative days. Early helmet therapy initiation is facilitated by routine administration of 24 hours of intravenous dexamethasone to minimize edema. We recommend that the helmet be worn for 23 hours per day until the age of 12 months. All patients treated with EAC received postoperative helmet therapy from two major orthotic companies (Hanger Clinic and Orthotic \& Prosthetic Lab, Inc.).

Our standard practice for postoperative follow-up after open CVR is one clinic visit at 3-6 weeks postoperatively and an additional visit at 1 year. Following EAC, patients are typically seen once at 3 weeks postoperatively, with frequency of subsequent visits determined by our familiarity with the treating cranial orthotist, as well as the patient's distance from our center. Patients meet with the orthotist every 2-3 weeks until the age of 6 months, then monthly until completion of helmet therapy. Multiple helmets may be required, and each new helmet incurs a charge, but there is no additional cost associated with orthotist visits for helmet adjustments. Most patients use a local orthotist, and we have implemented a telemedicine system for patients residing long distances from our center to reduce travel burden. Orthotic measurements are sent to the surgeon, and the patient and family communicate with us via telemedicine to follow helmet tolerance and head shape progression. Patients treated by less experienced orthotists are seen more frequently in person in our clinic for close monitoring of orthotic therapy. 
TABLE 1. Summary of patient characteristics and treatment charges

\begin{tabular}{|c|c|c|c|c|}
\hline Characteristic & Open Surgery & Endoscopic Surgery & Total & $p$ Value \\
\hline No. of patients & 41 & 38 & 79 & \\
\hline Mean age at op in mos* & $8.7 \pm 1.9$ & $3.5 \pm 1.6$ & NA & $<0.001$ \\
\hline \multicolumn{5}{|l|}{ Synostosis type, no. (\%) } \\
\hline Metopic & $20(48.8 \%)$ & $19(50.0 \%)$ & $39(49.4 \%)$ & \\
\hline Unicoronal & $19(46.3 \%)$ & $12(31.6 \%)$ & $31(39.2 \%)$ & \\
\hline Lambdoid & $2(4.9 \%)$ & $7(18.4 \%)$ & $9(11.4 \%)$ & \\
\hline Total & 41 & 38 & 79 & \\
\hline \multicolumn{5}{|c|}{ No. of clinic visits in 1st year } \\
\hline Mean* & $2.0 \pm 1.4$ & $4.0 \pm 2.2$ & NA & $<0.001$ \\
\hline Median & 2.0 & 3.5 & NA & \\
\hline \multicolumn{5}{|l|}{ Periop charges* } \\
\hline Hospital fees & $\$ 66,652 \pm \$ 16,206$ & $\$ 23,695 \pm \$ 6,732$ & NA & $<0.001$ \\
\hline Surgeon fees & $\$ 25,054 \pm \$ 9,577$ & $\$ 14,489 \pm \$ 2,041$ & NA & $<0.001$ \\
\hline Anesthesia fees & $\$ 3,482 \pm \$ 449$ & $\$ 1,784 \pm \$ 168$ & NA & $<0.001$ \\
\hline Mean helmet charges* & NA & $\$ 10,072 \pm \$ 4,059$ & NA & \\
\hline Total charges $\dagger$ & & & NA & \\
\hline Mean* & $\$ 95,588 \pm \$ 20,866$ & $\$ 50,840 \pm \$ 8,733$ & NA & $<0.001$ \\
\hline
\end{tabular}

\section{Data Collection}

Patient characteristics including diagnosis, age at surgery, length of stay (LOS), and insurance status were noted. Hospital financial records were reviewed for inpatient charges associated with the perioperative hospitalization, accounting for inpatient bed utilization, medical equipment, laboratory services, medications, and operating room supplies. Surgeon fees and associated Current Procedural Terminology (CPT) codes were collected, and anesthesia fees were noted. Outpatient records were reviewed for the number of postoperative clinic visits in the 1st year, with cost per visit estimated at \$200 to account for travel expenses and parental missed time from work..$^{39}$ For patients treated with EAC, data were collected from orthotic companies for number of helmets required per patient and charge per helmet. CPT coding data were collected for each patient. ${ }^{2}$

\section{Statistical Analysis}

The means, standard deviations, and interquartile ranges were calculated for age at cranial repair, inpatient charges, physician fees, outpatient clinic visits, and helmet therapy charges, as well as cumulatively for total charges in the 1st year of treatment. All charges were adjusted for inflation to reflect 2018 dollars (https://www. minneapolisfed.org/community/financial-and-economiceducation/cpi-calculator-information/consumer-priceindex-and-inflation-rates-1913). Distributions were found to be nonnormal; Mann-Whitney U-tests were performed to compare distributions of the two-subject groups on the aforementioned parameters. Bivariate correlations of age at surgery, LOS, inflation-adjusted total treatment charges, helmet charges, and number of helmets with date of repair were analyzed with Spearman's rho. Fisher's exact test was used to compare proportions. To compare the distributions of diagnoses between the subject groups, a script (G. Cardillo, MyFisher; https://www.mathworks. com/matlabcentral/fileexchange/26883-myfisher) was run in Matlab R2017 (MathWorks), and the mid-p correction value was reported. All other analyses were executed with SPSS version 23 (IBM Corp.).

\section{Results}

We identified 79 patients who met our inclusion criteria- 41 treated by CVR and 38 treated by EAC with postoperative helmet therapy. Patient characteristics and a summary of perioperative charges, cost of outpatient clinic visits, and helmeting charges are shown in Table 1. There was no statistically significant difference in the distribution of synostosis diagnoses between the two groups $(\mathrm{p}=0.112)$. The mean age at surgery of patients in the EAC group was 3.5 months, whereas in the CVR group it was 8.7 months $(\mathrm{p}<0.001)$. Neither the EAC $\left(r_{s}=-0.13, \mathrm{p}=0.439\right)$ nor the open CVR $\left(r_{s}=0.03, \mathrm{p}=0.872\right)$ group had a significant correlation between age and date of surgery (Fig. 1).

The median LOS was 3 days for the open group (range 2-5 days) and 1 day for the endoscopic group (range 1-2 days). Only 1 patient in the endoscopic group (treated in 2008) stayed for 2 days postoperatively. Figure 2 shows the trends in LOS over time. There was a moderate negative correlation between LOS and date of surgery in the open group $\left(r_{s}=-0.54, \mathrm{p}<0.001\right)$, with a trend toward decreasing LOS, and there were no stays longer than 3 days after 2014. 


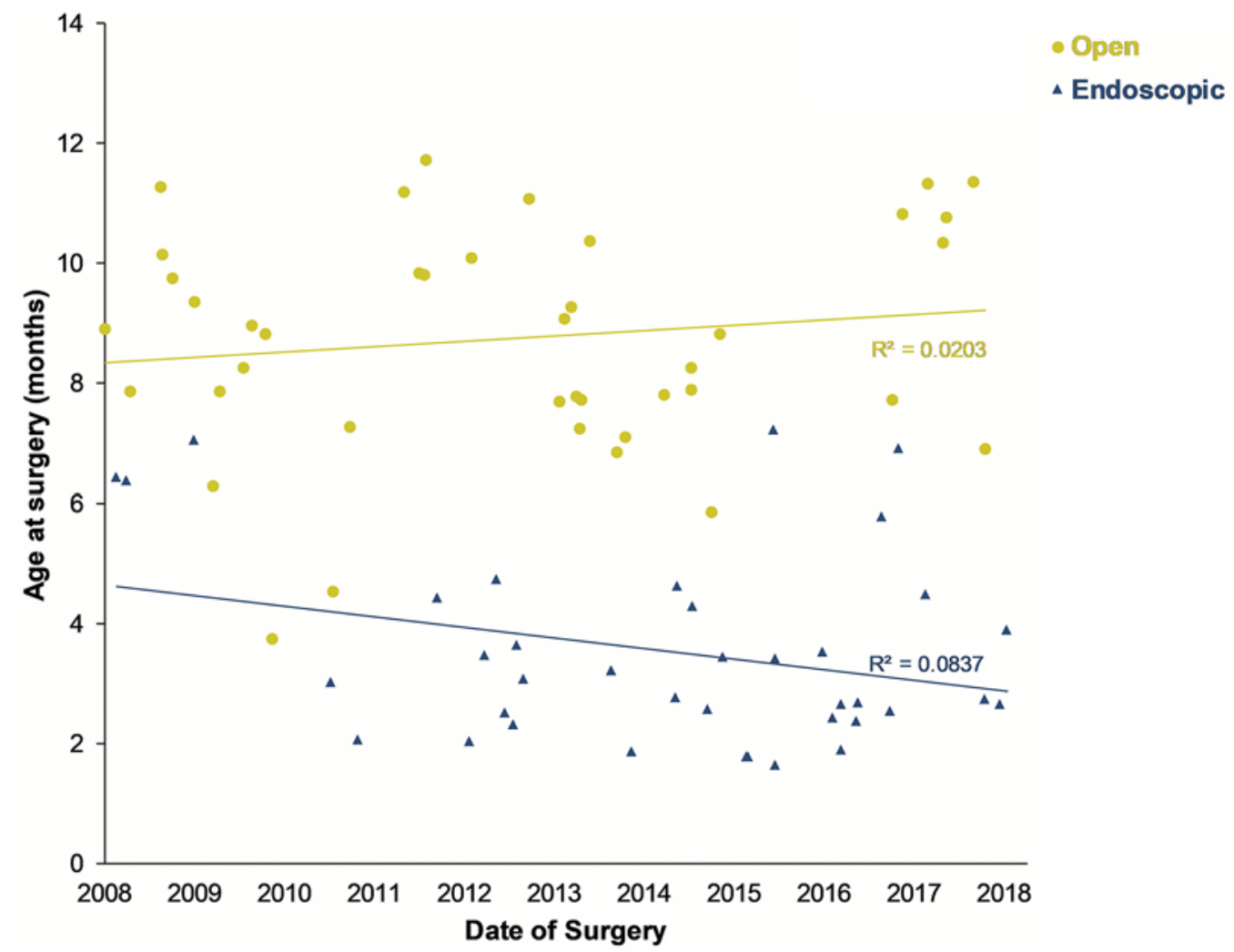

FIG. 1. Trends in age at surgery over time. Figure is available in color online only.

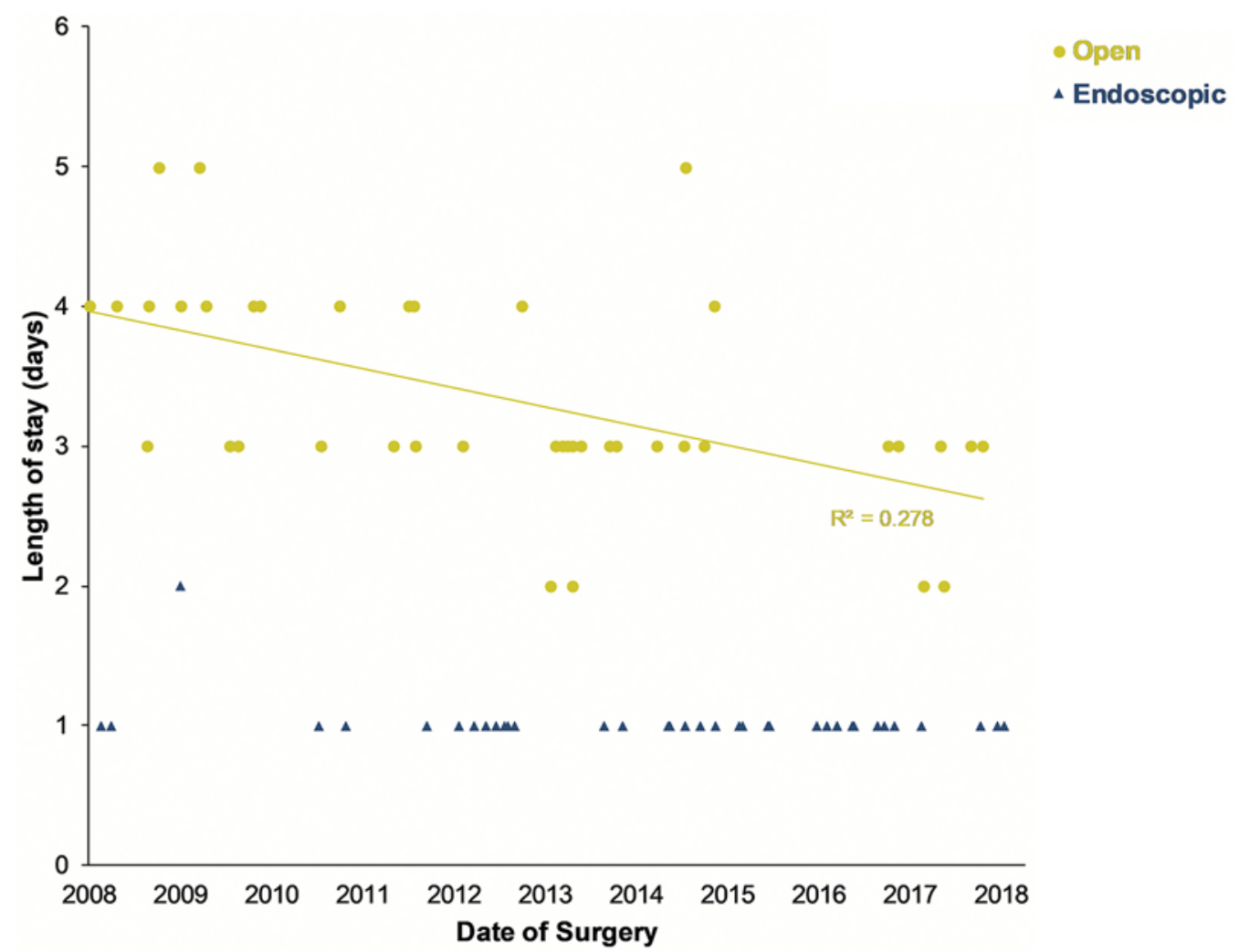

FIG. 2. Trends in LOS over time. Figure is available in color online only. 


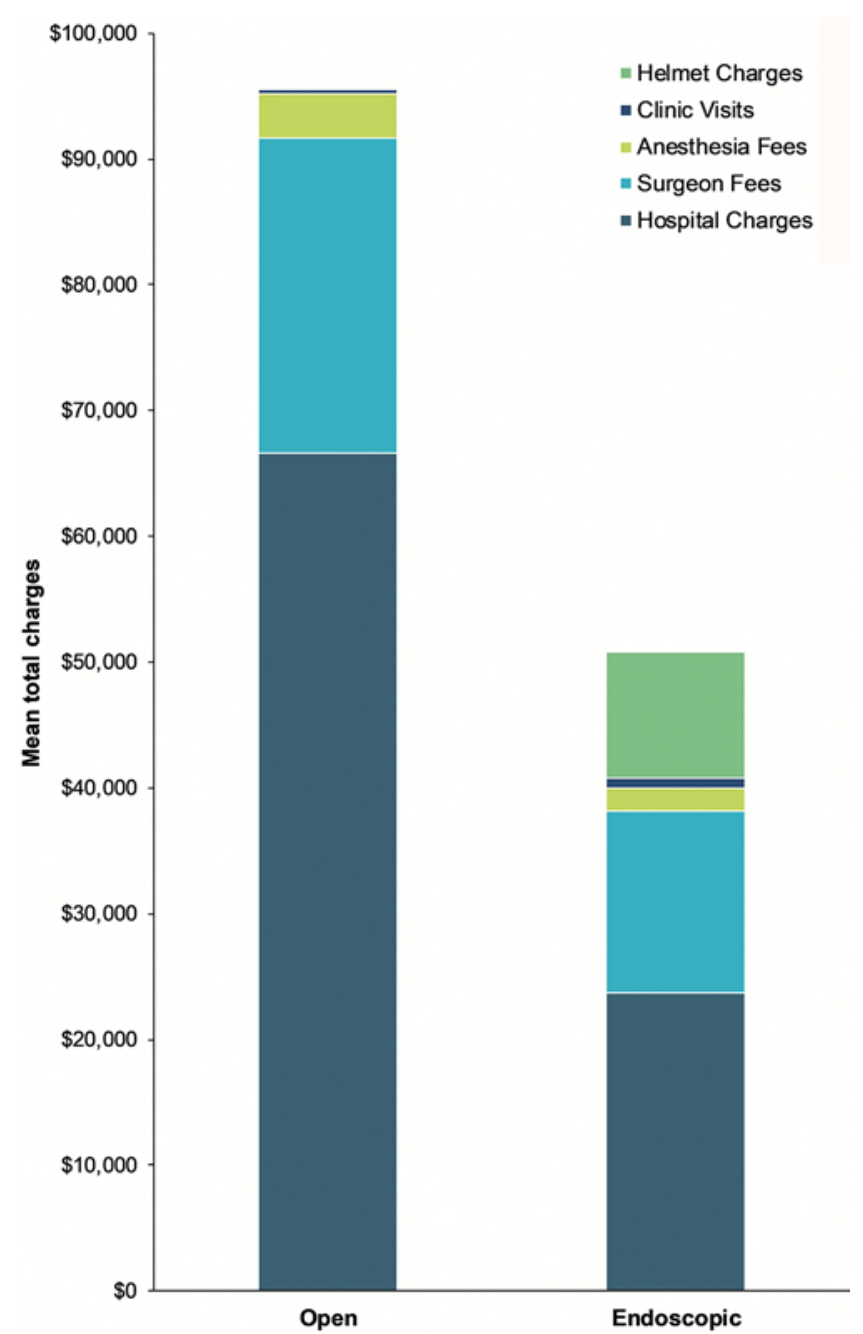

FIG. 3. Mean values for total charges of treatment in the 1st year are shown, as the sum of hospital, surgeon, and anesthesia fees, clinic visit costs, and helmet charges (for the endoscopic group). The mean charges for the 1st year of treatment for open CVR patients were significantly higher than those for EAC patients ( $\$ 95,588$ vs $\$ 50,840 ; p<0.001)$. Figure is available in color online only.

In both the EAC and CVR cohorts, hospital fees made up the largest proportion of perioperative charges, followed by surgeon fees and anesthesia fees. Mean total treatment charges were significantly lower for EAC than they were for open CVR $(\$ 50,840 \pm \$ 8733$ vs $\$ 95,588 \pm$ $\$ 20,866$; p < 0.001) (Fig. 3). Patients in the EAC cohort had significantly more clinic visits in the 1 st year than the patients in the CVR group (4 vs 2 visits; $p$ < 0.001) (Fig. 4). Figure 5 illustrates the trend toward increasing total charges for treatment over time. There was a strong positive correlation between charges for both open CVR $\left(r_{s}=\right.$ $0.81, \mathrm{p}<0.001)$ and $\operatorname{EAC}\left(r_{s}=0.61, \mathrm{p}<0.001\right)$ and date of surgery.

The number of helmets required for each patient in the EAC group was variable: $13 \%$ of patients required 1 helmet, $30 \%$ required 2 helmets, $40 \%$ required 3 helmets, and $13 \%$ required 4 or more helmets, with mean total helmeting charges of $\$ 10,072$. The trend toward increasing total charges for helmet therapy over time is shown in Fig. 6 , with a moderate positive correlation between helmet charges and date of surgery $\left(r_{s}=0.53, \mathrm{p}<0.001\right)$. Figure 7 shows the trend toward increased number of helmets per patient over time, again with a moderate positive correlation between number of helmets and date of surgery $\left(r_{s}=\right.$ $0.53, \mathrm{p}<0.001)$.

One patient in the open CVR group and no patients in the EAC group underwent reoperation before age 5 years (19 [50\%] of 38 EAC patients and 34 [83\%] of 41 CVR patients had reached the age of 5 years at the time of analysis]. This was a patient with metopic synostosis who had undergone FOA at 4 months of age and returned for cranioplasty with hydroxyapatite at 4.5 years of age to correct significant bitemporal narrowing and frontal prominence.

CPT coding was consistent between surgeons. All EAC procedures were filed under CPT code 21299-62 (unlisted craniofacial and maxillofacial procedure with 2-surgeon modifier). Open CVR for metopic and coronal synostosis was filed under CPT codes 21175 (reconstruction, bifrontal, superior-lateral orbital rims and lower forehead, advancement or alteration [e.g., plagiocephaly, trigonocephaly, brachycephaly], with or without grafts [included obtaining autografts]) and either 61557 (bifrontal bone flap) or 61559 (recontouring with multiple osteotomies and bone autografts [e.g., barrel-stave procedure] [includes obtaining grafts]). Open CVR for lambdoid synostosis was filed under CPT code 61559-62 (2-surgeon modifier).

The distribution of insurance types differed between the two groups: $41 \%$ of the open CVR cohort and 16\% of the EAC cohort were insured by Medicaid, and 59\% of the open CVR cohort and $84 \%$ of the EAC cohort were insured by private insurance $(\mathrm{p}=0.014)$. In a subgroup analysis of patients residing locally in the greater St. Louis metropolitan area (https://www2.census.gov/geo/maps/ metroarea/stcbsa_pg/Feb2013/cbsa2013_MO.pdf), this disparity was not significant. In this subgroup of 29 patients, there were 12 patients treated with open CVR and 17 patients treated with EAC: $17 \%$ (2/12) of the open CVR cohort and $12 \%(2 / 17)$ of the EAC cohort were insured by Medicaid, and $83 \%$ of the open CVR cohort and $88 \%$ of the EAC cohort were insured by private insurance ( $\mathrm{p}$ $=1.000$ ). There were no significant differences in total charges between privately and publicly insured patients for either procedure ( $p=0.812$ for open CVR, $p=0.544$ for $\mathrm{EAC})$.

\section{Discussion}

In the 2 decades since its introduction for the correction of sagittal synostosis, ${ }^{17}$ EAC with molding helmet therapy has gained traction as a viable treatment option for sagittal synostosis, but it is still not uniformly practiced across craniofacial centers for coronal, metopic, and lambdoid synostosis. The clinical advantages of endoscopic treatment of craniosynostosis over open CVR have been well documented and include decreased operative blood loss and transfusion requirements, as well as shorter operative times and LOS. 1,6,10,13,14,18,21,31,39 An increasing body of literature from our institution and others has demonstrated promising outcomes in cranial morphology after EAC 

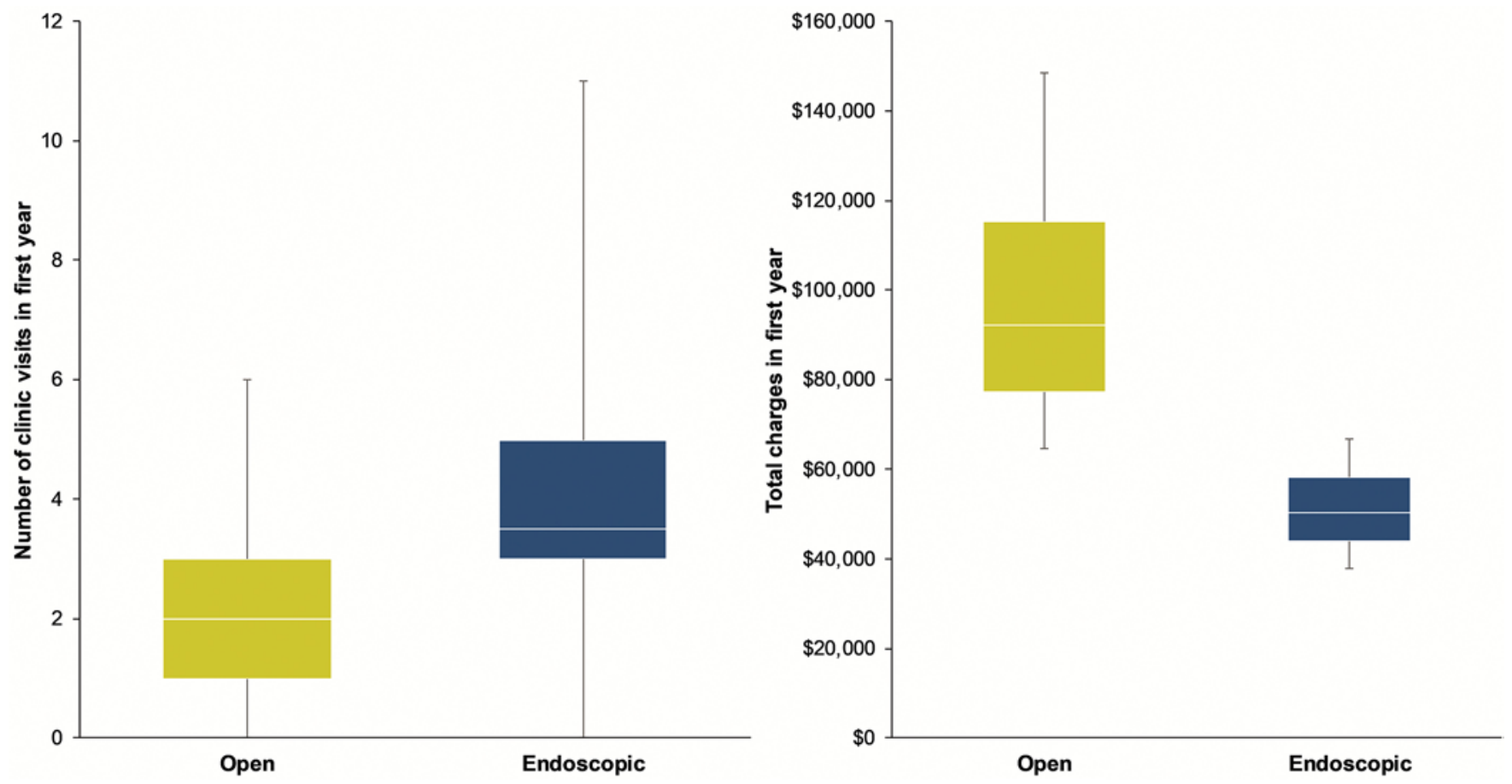

FIG. 4. Box-and-whisker plots comparing number of clinic visits (left) and total charges (right) for treatment in the 1st year between open CVR and EAC groups. Upper and lower box borders indicate first and third quartiles. Whiskers indicate maximum and minimum values. Median values are marked with a horizontal line within each box. Figure is available in color online only.

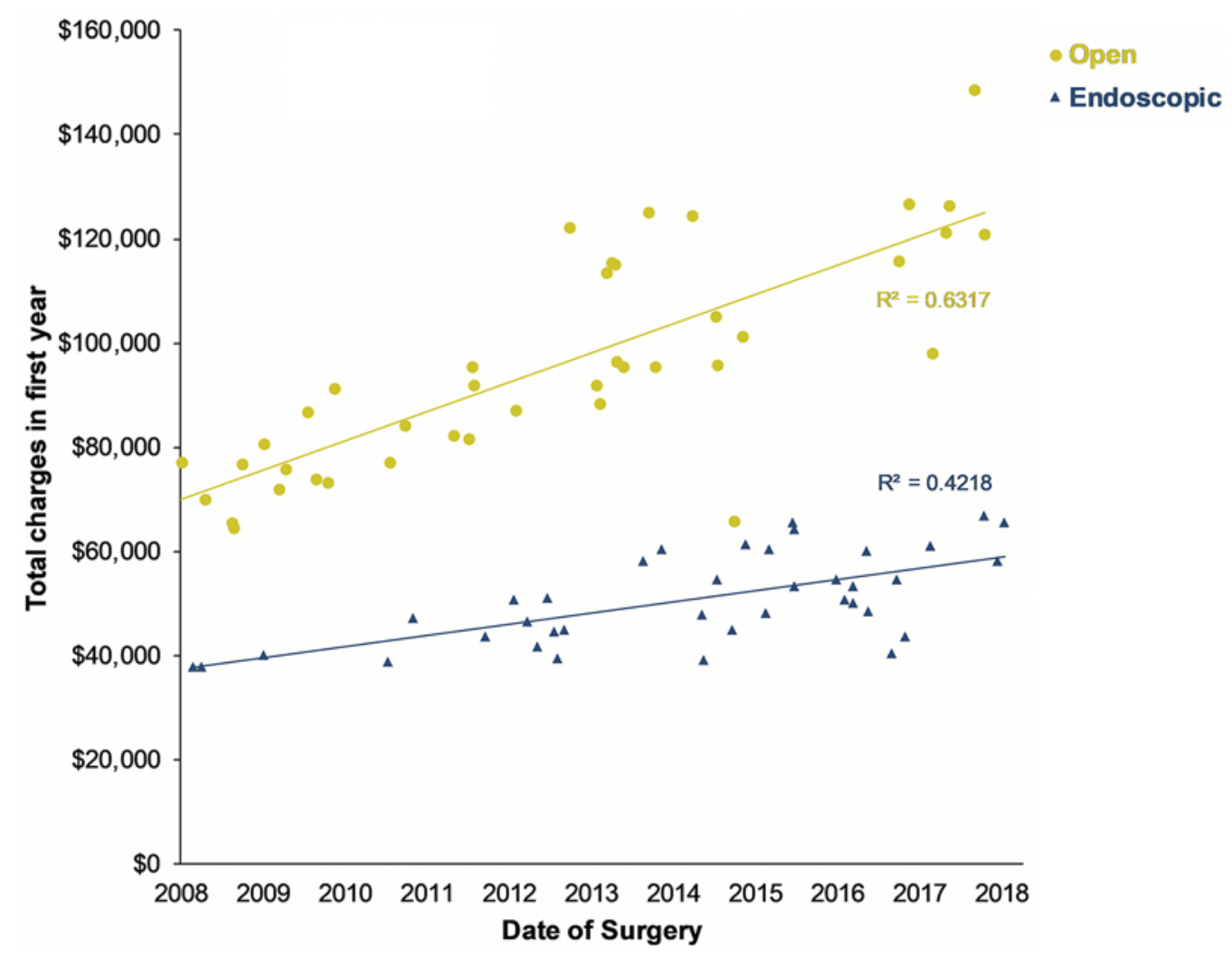

FIG. 5. Trend in total charges for treatment in the 1st year. Figure is available in color online only. 


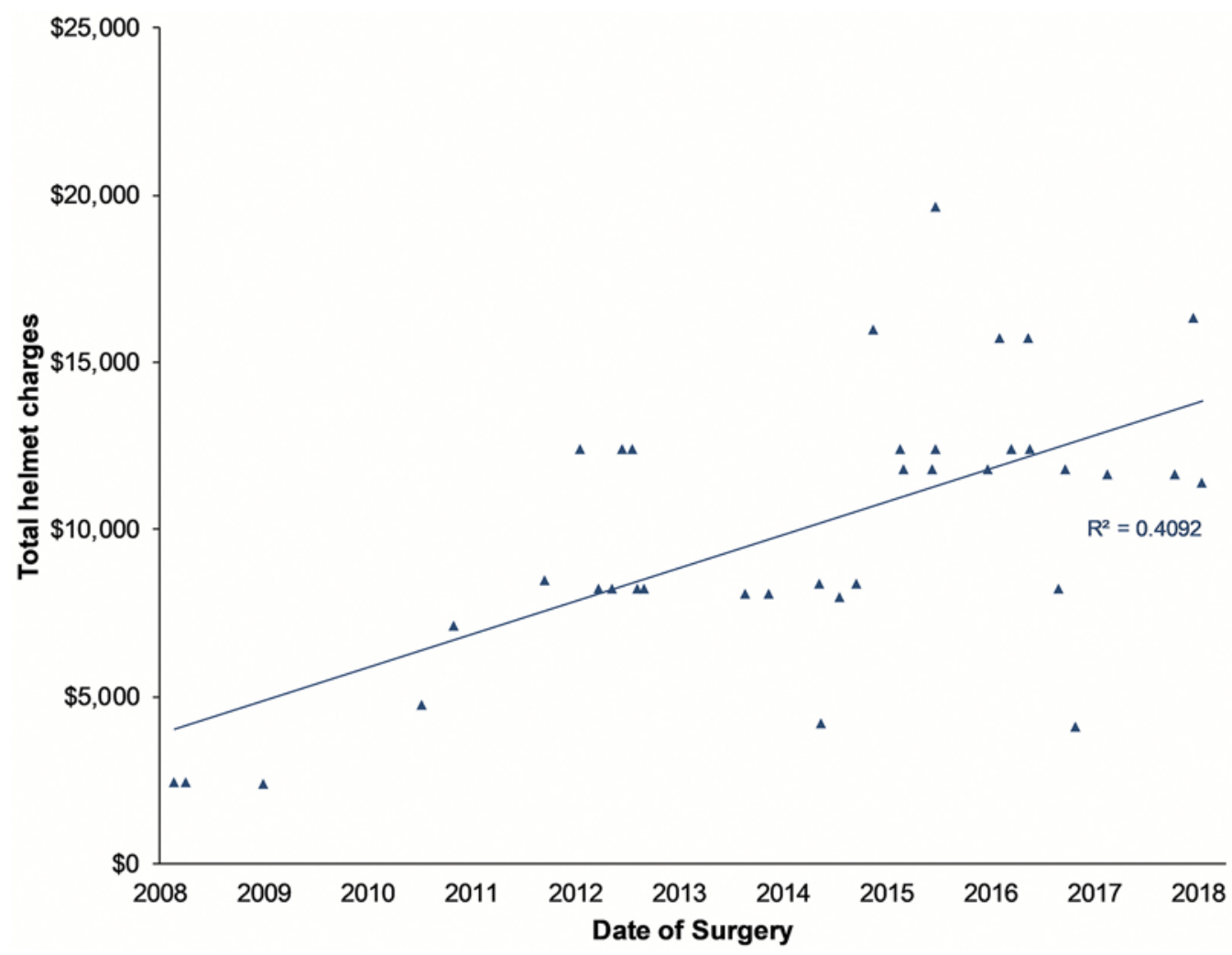

FIG. 6. Trend in helmet charges over time (endoscopic cohort only). Figure is available in color online only.

with helmet therapy, equal or superior to those of open CVR. $11,16,24,28,32,35,40,42$

With an increased focus on cost-effective healthcare delivery, previous studies have shown a significant decrease in financial burden with endoscopic treatment, most often demonstrated in patients with sagittal synostosis. ${ }^{1,8,19,23,31,39}$ Fewer data are available on the charges associated with surgical treatment of nonsagittal synostosis (metopic, coronal, and lambdoid), for which traditional open remodeling procedures are distinct from those for sagittal synostosis. Lambdoid synostosis is the least common of these 4 synostosis types, and there is a corresponding paucity of studies including these rare patients.

Two studies have reported cost comparisons between open and endoscopic treatments of nonsagittal synostosis but with significant limitations. Jivraj et al. recently reported a cost comparison for the treatment of unicoronal synostosis; because EAC is not practiced in the United Kingdom, the comparison was instead between data for open CVR (via FOA) performed at their center and data published by Jimenez and Barone for EAC performed in the United States, extrapolated to United Kingdomequivalent charges. ${ }^{16,19}$ Though the study shows data consistent with our experience for decreased charges with endoscopic treatment, the data are difficult to apply to the United States healthcare billing model. Chan et al. previously published a comparison of costs for a pooled cohort of patients with coronal, metopic, and sagittal synostosis, including syndromic and multisuture cases,${ }^{8}$ but the cost analysis was limited to the patients' hospital bills at dis- charge combined with helmeting costs for the endoscopic group. Surgeon fees were not included, and monetary values were not adjusted for inflation in a data set spanning a 10-year time period.

Our study presents a complete single-center analysis of a large cohort of 79 patients from the past 10 years, elucidating in detail the charges associated with FOA or posterior CVR versus an endoscope-assisted strip craniectomy for metopic, unicoronal, and lambdoid synostosis. We have included a comprehensive set of charges associated with each procedure beyond the inpatient hospital fees, including surgeon and anesthesia fees, the cost of outpatient clinic visits, and helmeting charges in the endoscopic group. Inflation-adjusted financial data allow for better direct comparisons in this longitudinal cohort.

We have also demonstrated a more drastic difference in charges between EAC and open CVR for patients with nonsagittal synostosis than what has previously been documented for sagittal synostosis. Vogel et al. demonstrated in 2014 that EAC confers an approximately $\$ 20,000$ savings over the 1st year in the treatment of patients with sagittal synostosis compared with open CVR. ${ }^{39}$ In the present study, EAC in the treatment of metopic, unicoronal, and lambdoid synostosis conferred an even greater mean savings of approximately $\$ 45,000$ when compared with conventional open treatment by FOA or posterior CVR, and the difference in charges between the two approaches has been increasing over time (Fig. 5). Contributing factors to this disparity may be the billing structure for CPT codes associated with FOA, which is a higher relative value unit 


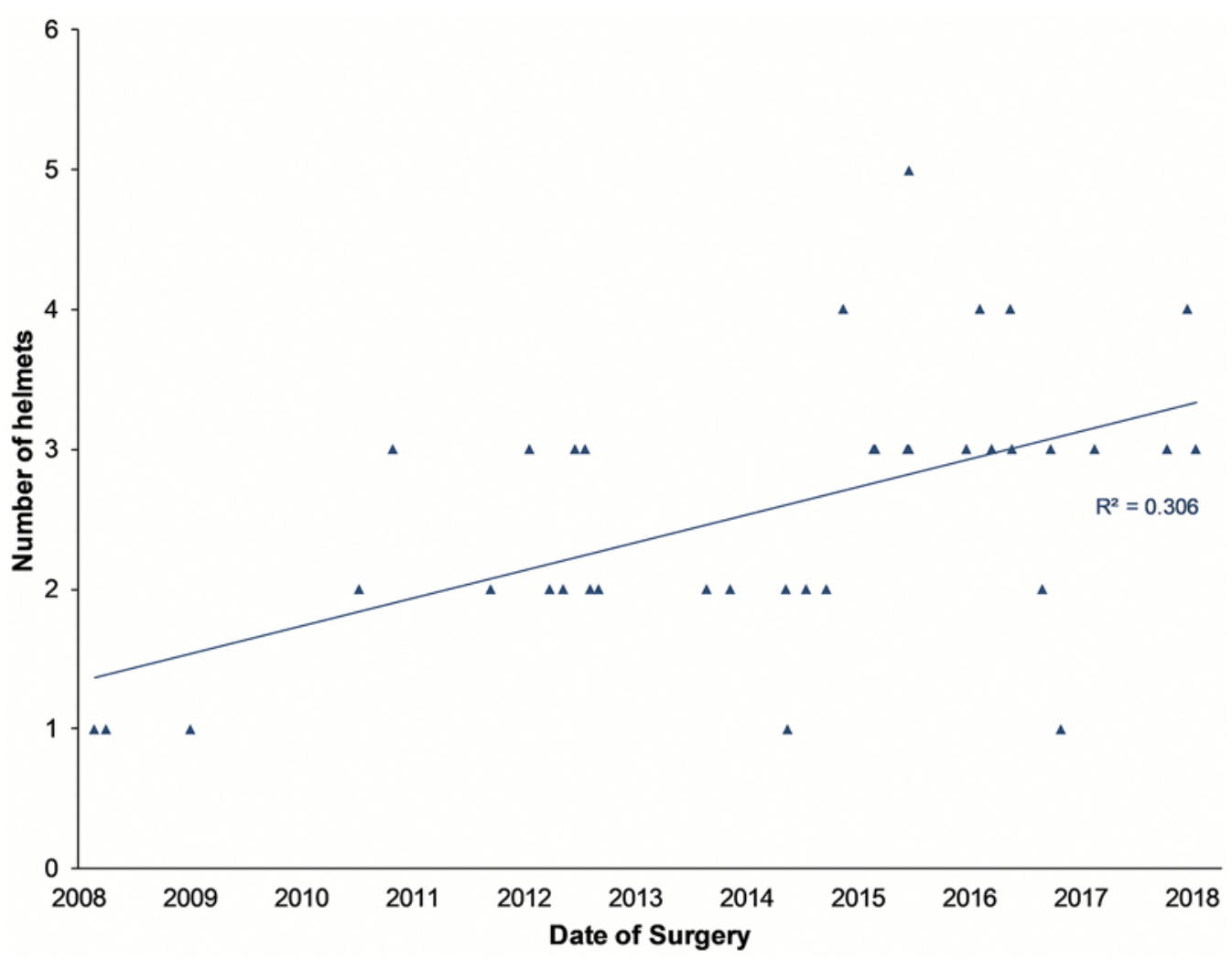

FIG. 7. Trend in number of helmets per patient over time (endoscopic cohort only). Figure is available in color online only.

procedure in craniofacial surgery, ${ }^{27}$ and the need for more hardware (absorbable plates and screws) in FOA compared to CVR for sagittal synostosis treated in children younger than 1 year of age.

We have noted a trend toward decreasing LOS in our open CVR patients, with no patients staying longer than 3 days postoperatively since 2014 . This coincided with our uniform implementation of postoperative intravenous dexamethasone treatment, which we have observed to markedly reduce edema formation. Despite these shorter hospital stays, the cumulative charges for open CVR have continued to rise over the years (Fig. 5).

While hospital charges make up the largest portion of the total charges in both open CVR and EAC, the significant charges for helmet therapy after EAC should be noted. Helmet therapy is an indispensable component of the endoscopic treatment strategy, and collaboration with an experienced orthotist is essential to achieving successful normalization of head shape. In the 10-year span of our data set, we have noted a trend toward a higher number of helmets per patient, translating to increasing overall charges for helmet therapy over time. The increase is due to our protocol of more aggressive head shape correction in the first few months of therapy, resulting in a shorter treatment period per helmet. In a changing insurance market that includes a trend toward high-deductible plans, ${ }^{4,15}$ this may be a consideration to address preoperatively with families, as some insurance plans may only provide partial coverage for helmet expenses. ${ }^{39}$ The majority of our center's publicly insured patients are insured by Illinois or
Missouri Medicaid, and both cover $100 \%$ of all postoperative helmet fees.

We offer both endoscopic and open CVR options to every eligible family regardless of their ability to pay, and while our data show that a lower proportion of patients in the endoscopic cohort were publicly insured compared to those in the open CVR cohort, the disparity was insignificant on subgroup analysis of patients residing locally. This may reflect a referral bias, with privately insured patients more likely to travel a distance seeking a second opinion or the option of endoscopic treatment, which is not available at all centers. The trend toward an increasing total price of treatment, even after adjustment for inflation, reflects the nationwide increase in healthcare spending in recent years. ${ }^{4,715}$ Though routine treatment of congenital conditions is arguably not the worst offender in ballooning healthcare costs in the US, it is prudent for physicians to understand the financial profile of each major procedure they offer.

The limitations of our study include the retrospective, single-center study design and the analysis of financial variables over a long study period. We have attempted to mitigate the latter with mathematical adjustments for inflation. The single-center design confers an advantage relative to other published studies, as discussed above, but it may limit the generalizability of our data to other centers, where treatment and hospitalization protocols may differ significantly, especially with regard to postoperative ICU care. Bonfield et al. ${ }^{5}$ and Wolfswinkel et al. ${ }^{41}$ have reported promising safety outcomes without routine postoperative 
ICU admission after open CVR, and elimination of ICU care may represent a significant reduction in overall hospital charges. The financial data available for our study do not specify the subgroups of charges comprising the overall hospital fee, and as such we cannot identify a single component responsible for the large difference in hospital fees between open and endoscopic treatments. Nevertheless, we recognize that ICU care is likely to be a significant contributor. We continue to routinely admit patients to the ICU for one night postoperatively after open CVR and would look forward to additional data on the safety of foregoing this practice prior to considering it as a costsaving measure.

The use of resorbable plating systems may also not be universal across centers. Alternative methods of fixation used by some surgeons include wire, nonabsorbable suture, and absorbable suture, which may bring down the perioperative charges for open CVR. ${ }^{12,20,36}$

We also recognize several limitations with respect to the calculation of overall costs. First, we used billed charges for hospital, anesthesia, and surgeon fees as a surrogate for true cost, which is the amount paid by the insurance company and/or patient. The actual amounts collected are not readily available, making charges the most practical substitute metric for these components of the overall cost. Second, travel expenses for orthotist visits were not included in this analysis because most of our patients used a local orthotist, and while the visits are free of charge and the financial burden of local travel is likely small relative to the total figures presented in this study, the time invested and potential wages lost for helmet adjustment visits are an additional component of the endoscopic treatment strategy that should be kept in mind when counseling patients. In addition, some states may not have comparable public insurance coverage of helmet therapy, which may significantly alter the costs to a family for endoscopic treatment at other centers. Third, while we accounted for estimated costs to the family for each outpatient clinic visit, the analysis did not include an estimate of opportunity costs for time spent away from home and work during the perioperative hospitalization. These costs would be most accurately measured with a prospective analysis.

We did not address complications in this cohort of patients. The cost of any complications requiring more frequent outpatient visits was captured in the analysis, but any complications requiring readmission were not included. Such complications are rare, and including the cost of a second admission or reoperation would not reflect the typical cost of treatment.

Finally, we observed a significant expected age difference between patients in the open CVR group and patients in the EAC group, which reflects our practice of offering EAC to a younger age group and the trend toward younger age at referral to our center in recent years. This may or may not directly affect the price of treatment, but reflects a sampling bias of the study.

Despite these limitations, our study illustrates a significant reduction in total charges for endoscopic treatment of metopic, coronal, and lambdoid synostosis relative to open CVR and adds valuable data to a literature dominated by studies of sagittal synostosis. Even with the significant additional charges for helmet therapy and more frequent outpatient visits, endoscopic treatment charges total an average of $\$ 45,000$ less than those for open CVR for patients with nonsagittal synostosis.

\section{Conclusions}

In conjunction with the evolving data on successful clinical outcomes for normalization of head shape, our data support EAC as a cost-minimizing treatment for eligible patients with metopic, coronal, and lambdoid synostosis. Future investigations of long-term morphological and cognitive outcomes will be essential to the evolution of craniosynostosis treatment.

\section{References}

1. Abbott MM, Rogers GF, Proctor MR, Busa K, Meara JG: Cost of treating sagittal synostosis in the first year of life. J Craniofac Surg 23:88-93, 2012

2. American Medical Association: Current Procedural Terminology (CPT) 2019 Professional Edition. Chicago: American Medical Association, 2019

3. Bennett KG, Bickham RS, Robinson AB, Buchman SR, Vercler CJ: Metopic craniosynostosis: a demographic analysis outside an urban environment. J Craniofac Surg 27:544547,2016

4. Bodenheimer T: High and rising health care costs. Part 1: seeking an explanation. Ann Intern Med 142:847-854, 2005

5. Bonfield CM, Basem J, Cochrane DD, Singhal A, Steinbok P: Examining the need for routine intensive care admission after surgical repair of nonsyndromic craniosynostosis: a preliminary analysis. J Neurosurg Pediatr 22:616-619, 2018

6. Braun TL, Eisemann BS, Olorunnipa O, Buchanan EP, Monson LA: Safety outcomes in endoscopic versus open repair of metopic craniosynostosis. J Craniofac Surg 29:856-860, 2018

7. Burns KM, Evans F, Pearson GD, Berul CI, Kaltman JR: Rising charges and costs for pediatric catheter ablation. $\mathbf{J}$ Cardiovasc Electrophysiol 24:162-169, 2013

8. Chan JW, Stewart CL, Stalder MW, St Hilaire H, McBride L, Moses MH: Endoscope-assisted versus open repair of craniosynostosis: a comparison of perioperative cost and risk. J Craniofac Surg 24:170-174, 2013

9. Elliott RM, Smartt JM Jr, Taylor JA, Bartlett SP: Does conventional posterior vault remodeling alter endocranial morphology in patients with true lambdoid synostosis? J Craniofac Surg 24:115-119, 2013

10. Esparza J, Hinojosa J: Complications in the surgical treatment of craniosynostosis and craniofacial syndromes: apropos of 306 transcranial procedures. Childs Nerv Syst 24:1421-1430, 2008

11. Farber SJ, Nguyen DC, Skolnick GB, Naidoo SD, Smyth MD, Patel KB: Anthropometric outcome measures in patients with metopic craniosynostosis. J Craniofac Surg 28:713-716, 2017

12. Fearon JA: Rigid fixation of the calvaria in craniosynostosis without using "rigid" fixation. Plast Reconstr Surg 111:2739,2003

13. Goyal A, Lu VM, Yolcu YU, Elminawy M, Daniels DJ: Endoscopic versus open approach in craniosynostosis repair: a systematic review and meta-analysis of perioperative outcomes. Childs Nerv Syst 34:1627-1637, 2018

14. Han RH, Nguyen DC, Bruck BS, Skolnick GB, Yarbrough $\mathrm{CK}$, Naidoo SD, et al: Characterization of complications associated with open and endoscopic craniosynostosis surgery at a single institution. J Neurosurg Pediatr 17:361-370, 2016 
15. Jen HC, Raval MV, Langham MR Jr, Calkins CM, Dasgupta $\mathrm{R}$, Shah SR, et al: Attitudes and beliefs of pediatric surgical specialists on costs of care and high deductible health plans. Am Surg 84:1410-1414, 2018

16. Jimenez DF, Barone CM: Early treatment of coronal synostosis with endoscopy-assisted craniectomy and postoperative cranial orthosis therapy: 16-year experience. J Neurosurg Pediatr 12:207-219, 2013

17. Jimenez DF, Barone CM: Endoscopic craniectomy for early surgical correction of sagittal craniosynostosis. J Neurosurg 88:77-81, 1998

18. Jimenez DF, Barone CM, Cartwright CC, Baker L: Early management of craniosynostosis using endoscopic-assisted strip craniectomies and cranial orthotic molding therapy. Pediatrics 110:97-104, 2002

19. Jivraj BA, Ahmed N, Karia K, Menon R, Robertson E, Sodha A, et al: A 24-month cost and outcome analysis comparing traditional fronto-orbital advancment and remodeling with endoscopic strip craniectomy and molding helmet in the management of unicoronal craniosynostosis: a retrospective bi-institutional review. JPRAS Open 20:35-42, 2019

20. Koh KL, Zain A: Surgical outcome after less "rigid" fixation in open cranial vault remodeling for craniosynostosis. J Craniofac Surg 29:861-867, 2018

21. Lee HQ, Hutson JM, Wray AC, Lo PA, Chong DK, Holmes $\mathrm{AD}$, et al: Analysis of morbidity and mortality in surgical management of craniosynostosis. J Craniofac Surg 23:1256-1261, 2012

22. Lee HQ, Hutson JM, Wray AC, Lo PA, Chong DK, Holmes $\mathrm{AD}$, et al: Changing epidemiology of nonsyndromic craniosynostosis and revisiting the risk factors. J Craniofac Surg 23:1245-1251, 2012

23. Liles C, Dallas J, Hale AT, Gannon S, Vance EH, Bonfield $\mathrm{CM}$, et al: The economic impact of open versus endoscopeassisted craniosynostosis surgery. J Neurosurg Pediatr 24:105-215, 2019

24. Masserano B, Woo AS, Skolnick GB, Naidoo SD, Proctor MR, Smyth MD, et al: The temporal region in unilateral coronal craniosynostosis: fronto-orbital advancement versus endoscopy-assisted strip craniectomy. Cleft Palate Craniofac J 55:423-429, 2018

25. Meara JG, Burvin R, Bartlett RA, Mulliken JB: Anthropometric study of synostotic frontal plagiocephaly: before and after fronto-orbital advancement with correction of nasal angulation. Plast Reconstr Surg 112:731-738, 2003

26. Natghian H, Song M, Jayamohan J, Johnson D, Magdum S, Richards P, et al: Long-term results in isolated metopic synostosis: the Oxford experience over 22 years. Plast Reconstr Surg 142:509e-515e, 2018

27. National Health Policy Forum: Relative value units (RVUs): the basics. (https://www.nhpf.org/library/the-basics/Basics_ RVUs_01-12-15.pdf) [Accessed November 18, 2019]

28. Nguyen DC, Farber SJ, Skolnick GB, Naidoo SD, Smyth MD, Kane AA, et al: One hundred consecutive endoscopic repairs of sagittal craniosynostosis: an evolution in care. J Neurosurg Pediatr 20:410-418, 2017

29. Nguyen DC, Patel KB, Skolnick GB, Naidoo SD, Huang AH, Smyth MD, et al: Are endoscopic and open treatments of metopic synostosis equivalent in treating trigonocephaly and hypotelorism? J Craniofac Surg 26:129-134, 2015

30. Patel KB, Skolnick GB, Mulliken JB: Anthropometric outcomes following fronto-orbital advancement for metopic synostosis. Plast Reconstr Surg 137:1539-1547, 2016

31. Ridgway EB, Berry-Candelario J, Grondin RT, Rogers GF, Proctor MR: The management of sagittal synostosis using endoscopic suturectomy and postoperative helmet therapy. J Neurosurg Pediatr 7:620-626, 2011

32. Rottgers SA, Lohani S, Proctor MR: Outcomes of endoscopic suturectomy with postoperative helmet therapy in bilateral coronal craniosynostosis. J Neurosurg Pediatr 18:281-286, 2016

33. Selber J, Reid RR, Chike-Obi CJ, Sutton LN, Zackai EH, McDonald-McGinn D, et al: The changing epidemiologic spectrum of single-suture synostoses. Plast Reconstr Surg 122:527-533, 2008

34. Smartt JM Jr, Reid RR, Singh DJ, Bartlett SP: True lambdoid craniosynostosis: long-term results of surgical and conservative therapy. Plast Reconstr Surg 120:993-1003, 2007

35. Tan SP, Proctor MR, Mulliken JB, Rogers GF: Early frontofacial symmetry after correction of unilateral coronal synostosis: frontoorbital advancement vs endoscopic strip craniectomy and helmet therapy. J Craniofac Surg 24:1190-1194, 2013

36. Thurston TE, Andrades P, Phillips RA, Ray PD, Grant JH III: Safety profile of wire osteosynthesis in craniosynostosis surgery. J Craniofac Surg 20:1154-1158, 2009

37. van der Meulen J: Metopic synostosis. Childs Nerv Syst 28:1359-1367, 2012

38. van der Meulen J, van der Hulst R, van Adrichem L, Arnaud E, Chin-Shong D, Duncan C, et al: The increase of metopic synostosis: a pan-European observation. J Craniofac Surg 20:283-286, 2009

39. Vogel TW, Woo AS, Kane AA, Patel KB, Naidoo SD, Smyth MD: A comparison of costs associated with endoscope-assisted craniectomy versus open cranial vault repair for infants with sagittal synostosis. J Neurosurg Pediatr 13:324-331, 2014

40. Williams CT, Segar DJ, Naidoo SD, Skolnick GB, Proctor MR, Smyth MD, et al: Evaluation of endoscopic strip craniectomy and orthotic therapy for bilateral coronal craniosynostosis. J Craniofac Surg 30:453-457, 2019

41. Wolfswinkel EM, Howell LK, Fahradyan A, Azadgoli B, McComb JG, Urata MM: Is postoperative intensive care unit care necessary following cranial vault remodeling for sagittal synostosis? Plast Reconstr Surg 140:1235-1239, 2017

42. Zubovic E, Woo AS, Skolnick GB, Naidoo SD, Smyth MD, Patel KB: Cranial base and posterior cranial vault asymmetry after open and endoscopic repair of isolated lambdoid craniosynostosis. J Craniofac Surg 26:1568-1573, 2015

\section{Disclosures}

Dr. Patel is a consultant for Stryker CMF and an educational speaker for Hanger Clinic. Dr. Naidoo is an educational speaker for Hanger Clinic.

\section{Author Contributions}

Conception and design: Patel, Zubovic, Skolnick, Smyth. Acquisition of data: Zubovic, Lapidus. Analysis and interpretation of data: Patel, Zubovic, Skolnick. Drafting the article: Zubovic. Critically revising the article: all authors. Reviewed submitted version of manuscript: all authors. Approved the final version of the manuscript on behalf of all authors: Patel. Statistical analysis: Zubovic, Skolnick. Study supervision: Patel, Smyth.

\section{Supplemental Information \\ Previous Presentations}

This research was presented in poster form at the 76th Annual Meeting of the American Cleft Palate-Craniofacial Association, Tucson, Arizona, April 11, 2019.

\section{Correspondence}

Kamlesh B. Patel: Washington University School of Medicine in St. Louis, MO. kamlesh.patel@wustl.edu. 